\title{
An Equivalent Electric Circuit for the Thermal Network of the Savona Campus Smart Polygeneration Microgrid
}

\author{
Luca Barillari, Stefano Bracco, Massimo Brignone, \\ Federico Delfino, Fabio Pampararo, Carola Pacciani, \\ Renato Procopio, Mansueto Rossi \\ Department of Naval, Electrical, and ICT Engineering, \\ University of Genova, \\ Genova, Italy \\ mansueto.rossi@unige.it
}

\author{
Alessandro Nilberto \\ Mechanical, Energy, Management and Transportation \\ Engineering Department \\ University of Genova \\ Genova, Italy
}

\begin{abstract}
The paper proposes a model for the thermal network of the Savona Campus Smart Polygeneration Microgrid (SPM) in terms of an equivalent electric circuit. Such model allows to represent in a simple but accurate way the behavior of the SPM thermal network in order to insert it into the Energy Management System (EMS) that is presently running in the SPM control room. The parameters of the thermal circuit are identified by means of an algorithm that minimizes the difference between the temperature profile as calculated with the circuit and the measured one.
\end{abstract}

Index Terms-- Energy Management, Optimization Algorithm, Parameter Identification, Smart grids, Thermal Network.

\section{INTRODUCTION}

One of the main focuses of microgrids research has been oriented to the development of optimal operational strategies that allow to exploit the maximum potentials of their promising features in the energy management context. Such strategies are typically implemented in the so-called Energy Management Systems (EMS).

Due to their importance, EMSs have been deeply investigated in various forms and architectures [1-2]. The most common architecture for EMSs is the multilevel hierarchical one [3], in which the higher level concerns the dispatch of active and reactive power of the programmable production units, while the lower ones account for a more detailed control of the single components. The highest level of this architecture recalls the principles of optimization of traditional electricity transmission networks, achieved by classical Optimal Power Flow (OPF) algorithms. However, the presence of dynamic components like storage devices together with the uncertainties that characterize the load request and the Renewable Energy Sources (RES) and the combined presence of electric and thermal infrastructures makes such optimization problem a very hard task from a computational point of view. To overcome this difficulty, many different papers have been published in literature with the aim of developing accurate and efficient algorithms for the microgrid
EMSs, addressing either the integration of RES rather than storage devices, or the so-called Demand Side Management (DSM), or again the satisfaction of electric network constraints [4-6].

In a recent paper [7], the attention of the authors has been concentrated on an efficient electric network modeling for optimization purpose that has reached a good compromise between accuracy and reduction in the CPU effort. However, the model of the thermal network consisted only of a pure thermal power balance equation, which is quite unrealistic as it is based on the knowledge of the thermal load request expressed in terms of a thermal power profile rather than a temperature one and does not consider the delay time between the instant in which the thermal source is switched on and the one in which the temperature reaches the desired value. The proposed paper aims at bridging this gap and basically defines an equivalent electric circuit for the SPM thermal network that is able to account for both these issues.

In literature, many studies deal with the modeling of thermal networks from different point of views, which are focused on optimization or simulation aspects. The advantages of district heating networks based on Combined Heating and Power (CHP) technologies have been shown in [8-9] and, in particular in [8], a model for the optimal daily operation of a district system with a CHP plant has been proposed.

As highlighted by Larsen et al. [10], models including a full physical representation of the thermal networks, in terms of mass flow rates, pressure drops and pipe dimensions, are computationally intensive and, as a consequence, they are not suitable to be inserted into EMSs; to this end, they propose a simplified method which permits to study a district heating network by reducing its topological complexity. On the other hand, Kuosa et al. [11] focus the attention on the differences between "Y" and "ring" networks and compare a traditional network with a ring one characterized by an innovative flow rate control method; furthermore, the same authors introduce [12] an equivalent thermal circuit to represent the heat transfer 
process in a heat exchanger and apply this methodology to study a district heating system.

More simplified models of thermal networks are usually implemented within Mixed-Integer Linear Programming (MILP) tools [13, 14, 15], where district heating networks are optimally designed and operated in order to minimize different objectives, such as capital and daily operational costs. In the aforementioned models, energy balance equations, operational constraints and correlations for the calculation of heat losses and pressure drops are reported.

The equivalent electric circuit for the SPM thermal network, proposed in the present contribution, represents a general approach that can be adopted to study heat distribution systems in a simplified but effective way; moreover, the model can be inserted into models used to optimize distributed generation units.

As will be detailed in the following, the parameters of the proposed equivalent electric circuit have been identified by means of an inverse procedure that, starting from input and output measurements, has found out the optimal values of such parameters minimizing the distance between the measured parameters time profile and the calculated one.

\section{THE THERMAL NETWORK OF THE SAVONA CAMPUS SMART POLYGENERATION MICROGRID}

In this section, a brief description of the SPM is provided (see [7] and [16] for details), also referring to the thermal network.

From the electric point of view, the microgrid is fed by a secondary substation $15 \mathrm{kV} / 400 \mathrm{~V}$; from this substation two feeders supply the loads of the Campus and the other two connect the main four SPM switchboards. As shown in Fig. 1, the main technologies connected within the SPM network are:

- three CSP (Concentrating Solar Power) units (electrical power $1 \mathrm{~kW}$, thermal power $3 \mathrm{~kW}$ each), coupled with Stirling engines;

- an $\mathrm{H}_{2} \mathrm{O} / \mathrm{LiBr}$ absorption chiller having a cooling rated power of $70 \mathrm{~kW}$;

- an electrical storage (SoNick batteries by FIAMM S.p.A) characterized by a rated capacity of $141 \mathrm{kWh}$;

- a roof mounted photovoltaic field ( $80 \mathrm{~kW}$ of peak);

- two recharging stations for electric vehicles;

- three CHP (Combined Heat and Power) units (2 Capstone C65 and 1 Capstone C30 gas turbines);

- two gas boilers (total rated thermal power of about $1000 \mathrm{~kW})$.

The aforementioned systems are controlled and monitored from a control room, where the servers running the EMS are installed, together with clients for operators. A double fiber optic ring connects the servers in the control room with the switches located in each switchboard, where TM 1703 ACP Remote Terminal Units (RTU) and I/O modules are installed.

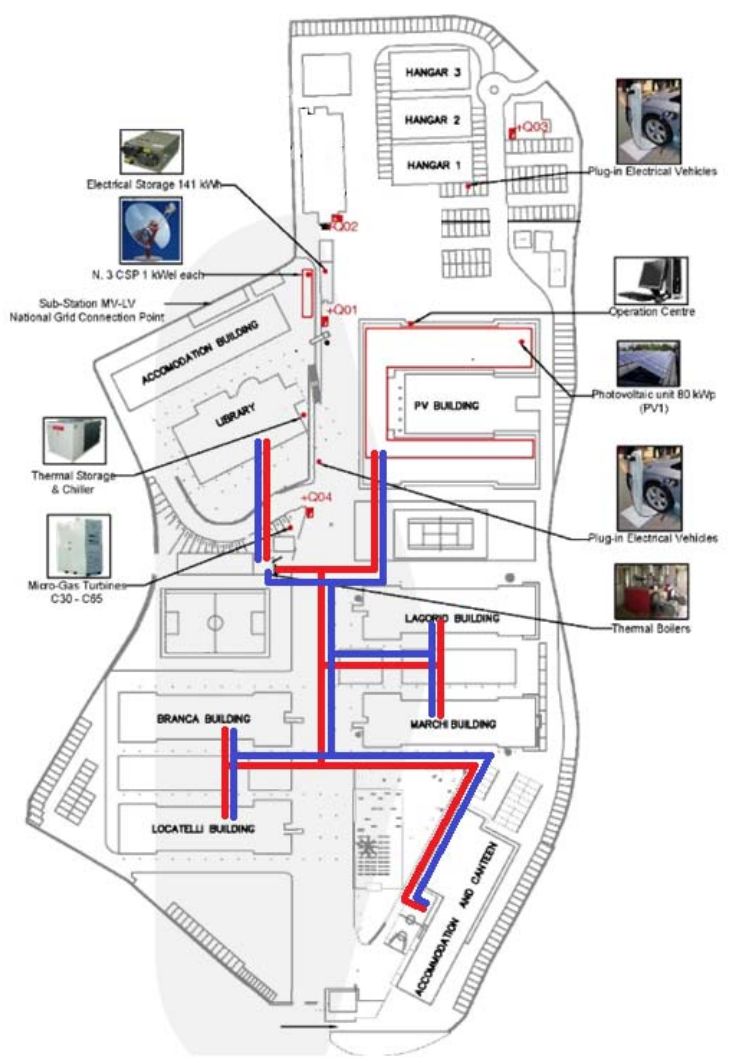

Figure 1. The SPM layout.

In Fig. 1 the SPM thermal network is sketched by red and blue lines, respectively corresponding to the supply lines, driving the hot water from the heating station to the users, and to the return lines, driving back the cold water from the users to the heating plants. The thermal network is fed by the three cogeneration gas turbines and the two gas boilers. The pipes are installed underground and are properly insulated in order to minimize heat losses; the pipe diameters are in the range between DN80 and DN125. The Fig. 1 does not show the pipes that distribute heat inside each building, where hot water is moved by on/off centrifugal pumps and heat is transferred to the indoor air by means of radiators or fan coils.

The heating network is real-time monitored from the control room by means of on-field sensors that allow measuring the following variables: the water mass flow rate, the supply and return lines temperatures, the thermal power output of each gas turbine and boiler and the outdoor temperature; at present, the buildings are not equipped with any sensor measuring the indoor conditions as well as the water temperature and flow rate, so that it is possible to evaluate only the whole thermal load of the Campus and not at a single building scale. In the next future, each building will 
be equipped with sensors in order to develop more accurate analyses and to highlight criticalities of the thermal network. In coldest winter days, the Campus thermal load can reach 900 $\mathrm{kW}$ of peak request and the thermal energy consumption is about $1300 \mathrm{MWh}$ per year.

\section{EQUIVALENT ELECTRIC CIRCUIT}

The aim of the paper is to develop a model for the Campus thermal network with the following features:

1. it has to be sufficiently simple in order to be put into the EMS without increasing too much the computational complexity;

2. it has to realistically model the delay time between the instant in which the thermal source is switched on and the one in which the temperature reaches the desired value;

3. it has to provide a relationship between the desired temperature time profiles and the corresponding thermal power that has to be produced by the three cogeneration gas turbines and the two gas boilers.

To do this, an equivalent electric circuit has been developed (Fig. 2) that contains resistors to model the thermal losses, capacitors that account for the thermal inertias, current sources that model the power generation and voltage sources that account for the external temperatures (ground and outdoor). Moreover, in order to accomplish feature 1), the circuit consists of only one thermal load representing the whole Campus (from here on denoted by "user"). In particular, referring to Fig. 2, one has that:

- the current source $I_{0}$ represents the thermal power generation (that can be produced either by the CHP units and/or by the boilers);

- the capacitance $C_{0}$ accounts for the thermal inertia responsible for the time delay necessary to obtain the desired temperature in the thermal circuit;

- $\quad$ the resistance $R_{0}$ models the thermal circuit losses;

- the voltage source $V_{0}$ models the fact that, without any thermal source, the thermal network temperature approaches the ground one;

- the switch $B_{1}$ models the possible disconnection between the thermal network and the load (encoded in a Boolean variable $\mathrm{S}$, true when $B_{1}$ is open);

- the diode $D_{1}$ models the fact that the user cannot transfer heat to the water in the thermal circuit;

- the resistance $R_{11}$ accounts for the pipe thermal losses in the thermal circuits of the buildings;

- the capacitance $C_{1}$ accounts for the thermal inertia responsible for the time delay necessary to obtain the desired temperature of the user;

- the resistance $R_{1}$ represents the thermal losses, that depends on the user insulation;

- the voltage $V_{1}$ mimics the fact that, without any thermal source, the user temperature approaches the outdoor ambient one.
In the proposed model, $V_{C 0}$ is the voltage between terminals $\mathrm{A}$ and $\mathrm{B}$ and represents the thermal network temperature, while $V_{C 1}$, which is the voltage between terminals $\mathrm{A}_{1}$ and $\mathrm{B}_{1}$, represents the user mean temperature.

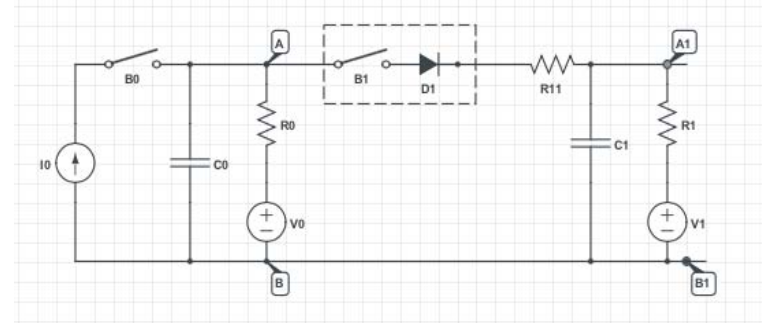

Figure 2. The SPM thermal network equivalent circuit.

Applying the Kirchoff's laws to the circuit of Fig. 2, one obtains the following set of two ordinary differential equations in the state variables $V_{C 0}$ and $V_{C 1}$ :

$$
\begin{array}{r}
\frac{d V_{C 0}(t)=-}{d t}=\frac{1}{C_{0}}\left(\frac{1}{R_{0}}+\frac{1}{R_{11}}\right) V_{C 0}(t)+\frac{1}{C_{0} R_{11}} V_{C 1}(t)+ \\
\quad+\frac{1}{C_{0}} I_{0}(t)+\frac{1}{C_{0} R_{11}} V_{B 1}(t)+\frac{1}{C_{0} R_{0}} V_{0}(t) \\
\frac{d V_{C 1}(t)}{d t}=\frac{1}{C_{1} R_{11}} V_{C 0}(t)-\frac{1}{C_{1}}\left(\frac{1}{R_{1}}+\frac{1}{R_{11}}\right) V_{C 1}(t)+ \\
\quad-\frac{1}{C_{1} R_{11}} V_{B 1}(t)+\frac{1}{C_{1} R_{1}} V_{1}(t)
\end{array}
$$

with the initial conditions

$$
\begin{aligned}
& V_{C 0}(t)=V_{C 0}^{*}, \\
& V_{C 1}(t)=V_{C 1}^{*} .
\end{aligned}
$$

Here the voltage $V_{B 1}$ at the switch $B_{1}$ is given by:

$$
V_{B 1}(t)=\left\{\begin{array}{cl}
V_{C 0}(t)-V_{C 1}(t) & \text { if } \mathrm{S}(t)=\text { true } \\
0 & \text { if } \mathrm{S}(t)=\text { false }
\end{array} .\right.
$$

The numerical solution of (1)-(2) allows to find out the time profile of both the thermal ring and the user temperatures.

\section{PARAMETER IDENTIFICATION}

Due to the complexity of the Campus thermal network, the numerical evaluation of the parameters appearing in the equivalent circuit starting from the physical properties of the network doesn't seem to be feasible. So, an identification procedure has been set up in order to find out the best values of such parameters that allow the proposed model to 
reproduce the time profile of the measured quantities with the highest accuracy. More precisely, once at disposal the measures $I_{0}, V_{0}$ and $V_{1}$ of in the time interval $[0, T]$, the optimal value of the parameters can be obtained by minimizing a weighted sum of the relative errors between the $V_{C 0}$ and its measure $V_{C 0 \text {,meas }}$, and the relative error between $V_{C 1}$ and its measure $V_{C 1 \text {,meas }}$ : i.e.

$$
\begin{gathered}
\min \left[p_{1} \frac{\int_{t=0}^{T}\left|V_{C 0}(t)-V_{C 0, \text { meas }}(t)\right| d t}{\int_{t=0}^{T}\left|V_{C 0, \text { meas }}(t)\right| d t}+\right. \\
\left.+p_{2} \frac{\int_{t=0}^{T}\left|V_{C 1}(t)-V_{C 1, \text { meas }}(t)\right| d t}{\int_{t=0}^{T}\left|V_{C 1, \text { meas }}(t)\right| d t}\right],
\end{gathered}
$$

subject to the obvious physical constraints that state that all the resistances and the capacitances must be positive. In (6) $p_{1}$ and $p_{2}$ represent two positive weights, such that

$$
p_{1}+p_{2}=1
$$

A numerical experiment has been conducted at the beginning of February at the Savona Campus, during a day when the thermal power was provided by the CHP units and the boilers. Moreover, three different timeslots can be defined as:

a) the building pumping system works from 7 a.m. to the 5 p.m.;

b) at 5 p.m. some loads are disconnected from the thermal network;

c) the overall thermal ring stops working at 10 p.m. and switches on again at 6 a.m.

This suggests the fact that the circuit topology changes during a typical day; as a consequence a time dependent behavior of $R_{0}$ and $C_{0}$ can be supposed: in a first approximation, piecewise constant functions are considered. More precisely,

$$
\begin{gathered}
R_{0}(t)=\left\{\begin{array}{cc}
R_{01} & \text { if } 7 \mathrm{am} \leq t<5 \mathrm{pm} \\
R_{02} & \text { if } 6 \mathrm{am} \leq t<7 \text { am or } 5 \mathrm{pm} \leq t<10 \mathrm{pm} \\
R_{03} & \text { if } 0 \mathrm{am} \leq t<6 \text { am or } 10 \mathrm{pm} \leq t \leq 12 \mathrm{pm}
\end{array}\right. \\
C_{0}(t)=\left\{\begin{array}{cc}
C_{01} & \text { if } 7 \text { am } \leq t<5 \mathrm{pm} \\
C_{02} & \text { if } 6 \mathrm{am} \leq t<7 \text { am or } 5 \mathrm{pm} \leq t<10 \mathrm{pm} \\
C_{03} & \text { if } 0 \mathrm{am} \leq t<6 \text { am or } 10 \mathrm{pm} \leq t \leq 12 \mathrm{pm}
\end{array}\right.
\end{gathered}
$$

\section{Moreover, condition $\mathrm{S}(t)$ becomes true if}

- 0 am $\leq t \leq 6$ am or $10 \mathrm{pm} \leq t \leq 12 \mathrm{pm}$, in order to encode condition c);

- $\quad V_{C 0}(t)>V_{C 1}(t)$, in order to encode the presence of the diode $\mathrm{D}_{1}$;

- $V_{C 1}(t) \leq V_{C 1, \max }(t)$, where $V_{C 1, \max }(t)$ is the user maximum temperature allowed at the instant time $t$.

In this framework, nine parameters have to be selected. It is possible to observe that in the time interval identified in c) the two networks (thermal circuit and user) are independent; moreover, since $I_{0}(t)=0$ in the same time period, the Cauchy problem can be solved analytically and in particular the solution depends only on the product $R_{03} C_{03}$. For this reason, the number of parameters to be estimated can be reduced to eight, by fixing $C_{03}=10^{8} \mathrm{~J} /{ }^{\circ} \mathrm{C}$, without losing in generality.

In the here presented test a period of 22 hours (from 11 am to 9 am of the day after), has been considered, during which the trend of $I_{0}, V_{0}$ and $V_{1}$ are reported in Fig. 3.
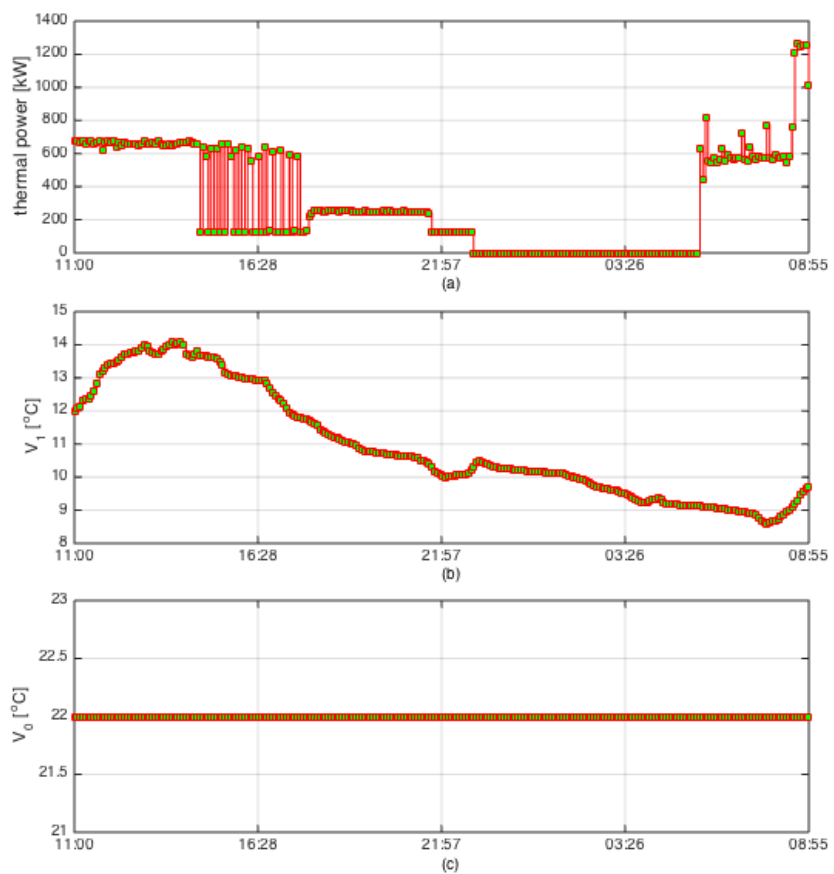

Figure 3. Time profile of the measured thermal power $I_{0}$ (panel a), the external temperature $V_{1}$ (panel b) and the ground temperature $V_{0}$ (panel c).

The parameter identification is carried out with an optimization method according to stochastic approach: the Ant Colony Optimization (ACO) algorithm [17]. The minimum value of the cost function (6), which is 0.03 , has been found for the value of the parameters reported in Table I. Moreover, by using those values of the parameters, the obtained trends of 
$V_{C 0}$ and $V_{C 1}$ have been superimposed to the measured ones (Fig. 4) showing a satisfactory agreement.

TABLE I. OPTIMAL PARAMETERS VALUES

\begin{tabular}{|c|c|c|}
\hline Parameter & Value & Units \\
\hline$R_{01}$ & $6.8610^{-5}$ & ${ }^{\circ} \mathrm{C} / \mathrm{W}$ \\
\hline$R_{02}$ & $1.1910^{-3}$ & ${ }^{\circ} \mathrm{C} / \mathrm{W}$ \\
\hline$R_{03}$ & $6.0510^{-4}$ & ${ }^{\circ} \mathrm{C} / \mathrm{W}$ \\
\hline$C_{01}$ & $4.3710^{8}$ & $\mathrm{~J} /{ }^{\circ} \mathrm{C}$ \\
\hline$C_{02}$ & $2.7710^{8}$ & $\mathrm{~J} /{ }^{\circ} \mathrm{C}$ \\
\hline$C_{03}$ & $1.0010^{8}$ & $\mathrm{~J} /{ }^{\circ} \mathrm{C}$ \\
\hline$R_{1}$ & $7.9710^{-4}$ & ${ }^{\circ} \mathrm{C} / \mathrm{W}$ \\
\hline$C_{1}$ & $1.1610^{8}$ & $\mathrm{~J} /{ }^{\circ} \mathrm{C}$ \\
\hline$R_{11}$ & $4.6510^{-4}$ & ${ }^{\circ} \mathrm{C} / \mathrm{W}$ \\
\hline
\end{tabular}
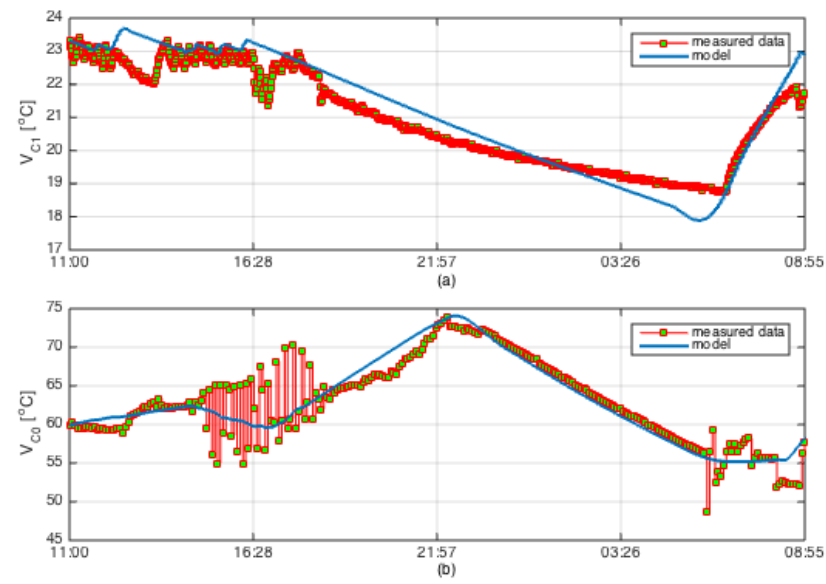

Figure 4. Result of the fitting. User temperature (panel a) and thermal network temperature (panel b).

In order to test the robustness of the procedure, the obtained values for the resistances and the capacitances have been used to reproduce the temperatures trend from the measurements of the thermal power in another day. Again, a period of 22 hours has been considered, and the trends of $I_{0}$, $V_{0}$ and $V_{1}$ as recorded during the further experimental acquisition are shown in Fig. 5. Once more, the obtained profiles are in accordance with the two measured temperatures (Fig. 6). Here, in fact, the value of the cost function resulted 0.06 .

\section{CONCLUSIONS}

The aim of this contribution has been to model the thermal network of the Savona Campus Smart Polygeneration Microgrid (SPM) in terms of an equivalent electric circuit that flexes its behavior. The values of the parameters involved in this model have been computed by means of an optimization procedure that minimizes the difference between the temperature profiles of the thermal circuit and the user as calculated with the proposed model and the measured ones.

The obtained numerical results show that the model describes in a very accurate way the set of measured temperatures, with an error of the order of three percent. Moreover, the robustness test highlighted a good fitting between the two profiles with a gap of only six percent.
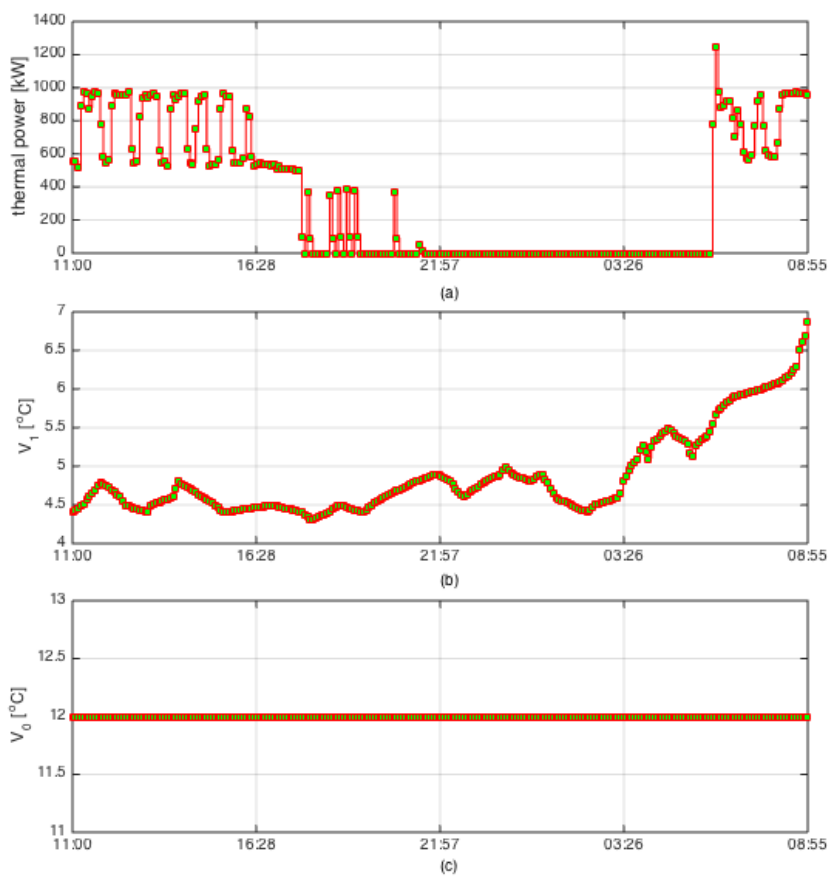

Figure 5. Time profile of the measured thermal power $I_{0}$ (panel a), the external temperature $V_{1}$ (panel b) and the ground temperature $V_{0}$ (panel c) in the consistency test.
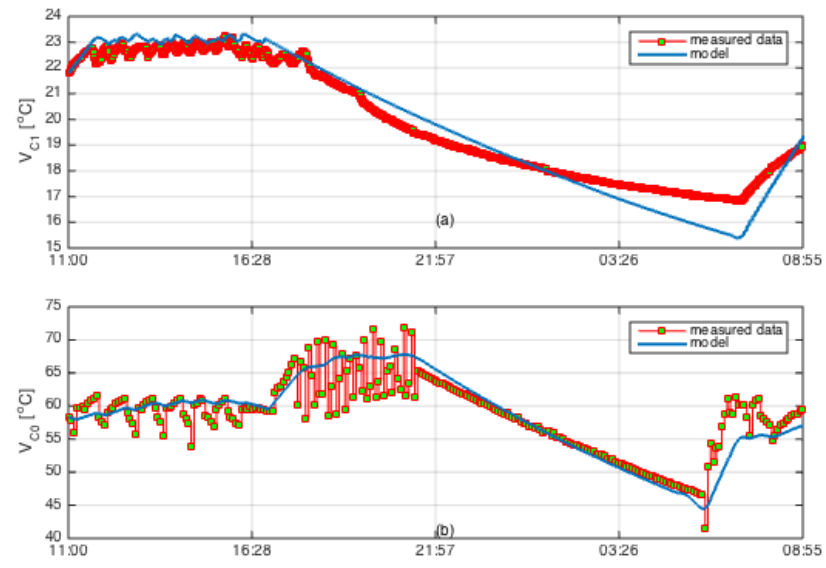

Figure 6. Results of the fitting in the consistency test. User temperature (panel a) and thermal network temperature (panel b). 


\section{REFERENCES}

[1] R. Palma-Behnke, C. Benavides, F. Lanas, B. Severino, L. Reyes, J. Llanos, D. Sáez, "A microgrid energy management system based on the rolling horizon strategy," IEEE Trans. Smart Grids., vol. 4, no. 2, pp. 996-1006, Jun. 2013.

[2] N. Hatziargyriou, "Microgrids - large scale integration of microgeneration to low voltage Grids" Paper C6-309 in Cigrè Biennial Section, Paris 2006.

[3] J. C. Vasquez, J. M. Guerrero, J. Miret, M. Castilla, and L. G. deVicuna, "Hierarchical control of intelligent microgrids," IEEE Ind. Electron. Mag., vol. 4, pp. 23-29, Dec. 2010.

[4] A. Bonfiglio, M. Brignone, F. Delfino, R. Procopio "Optimal Control and Operation of Grid-Connected Photovoltaic Production Units for Voltage Support in Medium Voltage Networks" IEEE Transaction on Sustainable Energy, vol. 5, no. 1, pp. 254-263, Jan. 2014.

[5] H. Nikkhajoei and R. Iravani, "Steady-state model and power flow analysis of electronically-coupled distributed resource units," IEEE Trans. Power Del., vol. 22, no. 1, pp. 721-728, Jan. 2007.

[6] P. Palensky and D. Dietrich, "Demand side management: demand response, intelligent energy systems and smart loads", IEEE Trans. Ind. Inf, vol 7, no. 3, pp. 381-388, Aug. 2011.

[7] S. Bracco, F. Delfino, F.Pampararo, M. Robba, and M. Rossi, "A mathematical model for the optimal operation of the University of Genoa Smart Polygeneration Microgrid: evaluation of technical, economic and environmental performance indicators," Energy, vol. 64, pp. 912-922, Jan. 2014.

[8] R. Aringhieri and F. Malucelli, "Optimal operations management and network planning of a district heating system with a combined heat and power plant", Annals of Operations Research, vol. 120, pp. 173-199, 2003.

[9] B. Rezaie and M.A. Rosen, "District heating and cooling: review of technology and potential enhancements", Applied Energy, vol. 93, pp. 2-10, 2012.

[10] H.V. Larsen, H. Palsson, B. Bohn, H.F. Ravn, "Aggregated dynamic simulation model of district heating networks", Energy Conversion and Management, vol. 43, pp. 995-1019, 2002.

[11] M. Kuosa, K. Kontu, T. Makila, M. Lampinen, R. Lahdelma, "Static study of traditional and ring networks and the use of mass flow control in district heating applications", Applied Thermal Engineering, vol. 54, pp. 450-459, 2013.

[12] Q. Chen, R.H. Fu, Y.C. Xu, "Electrical circuit analogy for heat transfer analysis and optimization in heat exchanger networks", Applied Energy, vol. 139, pp. 81-92, 2015.

[13] C. Haikarainen, F. Pettersson, H. Saxen, "An MILP model for distributed energy system optimization", Chemical Engineering Transactions, vol. 35, pp. 295-300, 2013.

[14] S. Bracco, G. Dentici, S. Siri, "Economic and environmental optimization model for the design and the operation of a combined heat and power distributed generation system in an urban area", Energy, vol. 55, pp. 1014-1024, 2013.

[15] T. Wakui, T. Kinoshita, R. Yokoyama, "A mixed-integer linear programming approach for cogeneration-based residential energy supply networks with power and heat interchanges", Energy, vol. 68, pp. 29-46, 2014.

[16] S. Bracco, F. Delfino, F. Pampararo, M. Robba, M. Rossi, "Planning and Management of Sustainable Microgrids: the Test-Bed Facilities at the University of Genoa", IEEE Africon 2013 Conference, Mauritius, pp. 1-5, 9-12 September 2013

[17] M. Dorigo and L.M. Gambardella, "Ant colony system: a cooperative learning approach to the travelling salesman problem," IEEE Trans. Evol. Comp., vol. 1, pp. 53-66, 1997. 